\title{
Chain length probability distribution - equivalence of ASYNNNI and 1D Ising model
}

Research Article

\author{
Mirjana Milić*
}

The "VINČA" Institute of Nuclear Sciences, Laboratory for Theoretical Physics and Condensed Matter physics 020, P.O. Box 52211001 Belgrade, Serbia

Received 7 November 2007; accepted 14 February 2008

\begin{abstract}
:
An expression for the chain length probability distribution $p(l)$ of a one dimensional Ising chain was derived using the cluster variation method formalism, the $p(l)$ being expressed through the pair cluster probabilities. It was shown numerically that the same expression also applies in the case of one dimensional chains formed along one of the next-nearest neighbor interactions included in the two dimensional ASYNNNI (Asymmetric Next-Nearest Neighbor Ising) model, widely used to describe the statistics of oxygen ordering in the basal $\mathrm{CuO}_{x}$ planes of the $\mathrm{YBa}_{2} \mathrm{Cu}_{3} \mathrm{O}_{6+x}$ type high- $\mathrm{T}_{C}$ superconducting materials. Equivalency between ASYNNNI and 1d Ising model is discussed.
\end{abstract}

PACS (2008): 64.60.De; 05.50.+q; 74.72.Bk

Keywords: Ising model $\cdot$ ASYNNNI model $\cdot$ probability distribution

(C) Versita Warsaw and Springer-Verlag Berlin Heidelberg.

\section{Introduction}

It is well known that the oxygen concentration, $x$, plays a significant role in determining the superconducting and structural properties of the high $\mathrm{T}_{c}$ superconductor, $\mathrm{YBa}_{2} \mathrm{Cu}_{3} \mathrm{O}_{6+x}$ (YBCO). Soon after the discovery of $\mathrm{YBa}_{2} \mathrm{Cu}_{3} \mathrm{O}_{6+x}$ it was established that when the oxygen content exceeds $x=0.35$, the oxygen atoms in the basal $\mathrm{CuO}_{x}$ planes (also referred as the chain plane) tend to arrange into $\mathrm{Cu}-\mathrm{O}$ chains of different lengths [1] aligned along one of the crystallographic axes, usually taken to be the $b$ axis. This chain formation initiates a structural phase transition from a disordered tetragonal state to an

*E-mail: mikac@vin.bg.ac.yu orthorhombic structural phase. The existence of five different orthorhombic phases has been established experimentally. The five orthorhombic phases are characterized by both differences in the way that sequences of $\mathrm{Cu}-\mathrm{O}$ chains are arranged along the $a$ axis, and differences in the unit cell periods [2]. The fact that only orthorhombic samples are superconducting, led to the conclusion that the formation of $\mathrm{Cu}-\mathrm{O}$ chains is crucial for the hole doping of the superconducting $\mathrm{CuO}_{2}$ planes which causes the superconducting behavior in YBCO $[3,4]$. Since the YBCO material becomes superconducting only after a certain oxygen stoichiometry is achieved, it was assumed that this coincides with oxygen chains reaching some critical length $l_{c r}$. The theory of critical chain length and the microscopic properties of the chain fragments were investigated in several theoretical studies [5-9]. The general conclusion of these studies was that only chains longer than a critical length, 
whose value was argued $[8,10]$ to be 3 or 4 , can transfer charge out of the basal planes, thus providing the increase in the number of the mobile charge carriers in the $\mathrm{CuO}_{2}$ sheets which leads to the appearance of superconductivity [11].

One of the first theoretical models proposed for the study of oxygen ordering in the basal planes of YBCO was the twodimensional Ising model with asymmetric interactions of next nearest neighbors (ASYNNNI model). The ASYNNNI model was originally developed by de Fontaine et al. [12] and, at the time, adequately described all the main features of the structural phase diagram and correctly predicted the order of the phase transitions. However, the model failed to stabilize other than the Ortho I and Ortho II (Fig. 1) orthorhombic structural phases. Though attempts have been made to extend the model in order to include other experimentally observed structures [13-16], the plain ASYNNNI model has remained the most widely used model for the investigation of the thermodynamics of oxygen ordering in the $\mathrm{CuO}_{x}$ planes.

Recently, the plain ASYNNNI model has been employed in extensive studies of the properties of the distribution of the $\mathrm{CuO}$ chains with respect to chain length $[17,18]$. It was found that the chain length probability distribution satisfies the simple geometrical law [17] i.e. that the distribution is determined by only one parameter. This parameter is the average chain length, $l_{a v}$, for a given oxygen sublattice and is experimentally measurable. This result was derived, in the case of low temperatures, by studying the structure of low energy levels and was confirmed in Monte Carlo numerical simulations for Ortho I and II structural phases. The result was confirmed for the Ortho II structural phase at a temperature of $T \approx 450 \mathrm{~K}$, where $T$ is the oxygen equilibrium temperature [18], and in the case of the Ortho I phase for temperatures as high as $T \approx 1800$ $K$ [17]. Furthermore, it was concluded that this specific form of the chain probability distribution should be valid for all oxygen concentrations, and all temperatures where the orthorhombic structures are stable, except in the close vicinity of the line of the critical points [18]. Since the same geometric chain length probability distribution also applies in the case of the infinite one dimensional Ising model $[19,20]$, the conclusion was made that the result obtained is another manifestation of the previously established low temperature equivalence between ASYNNNI and $1 \mathrm{~d}$ Ising model, in which the role of the Ising chain $\mathrm{NN}$ coupling $J$ is played by the $\mathrm{NNN}$ interaction $V_{2}$ of the ASYNNNI model [21].

In this work we will use the cluster variation method (CVM) formalism, to show that the chain length probability distribution $p(l)$ of the $1 \mathrm{~d}$ lsing model can be expressed through the pair cluster probabilities $x_{i}$ ( $i$ denotes one of
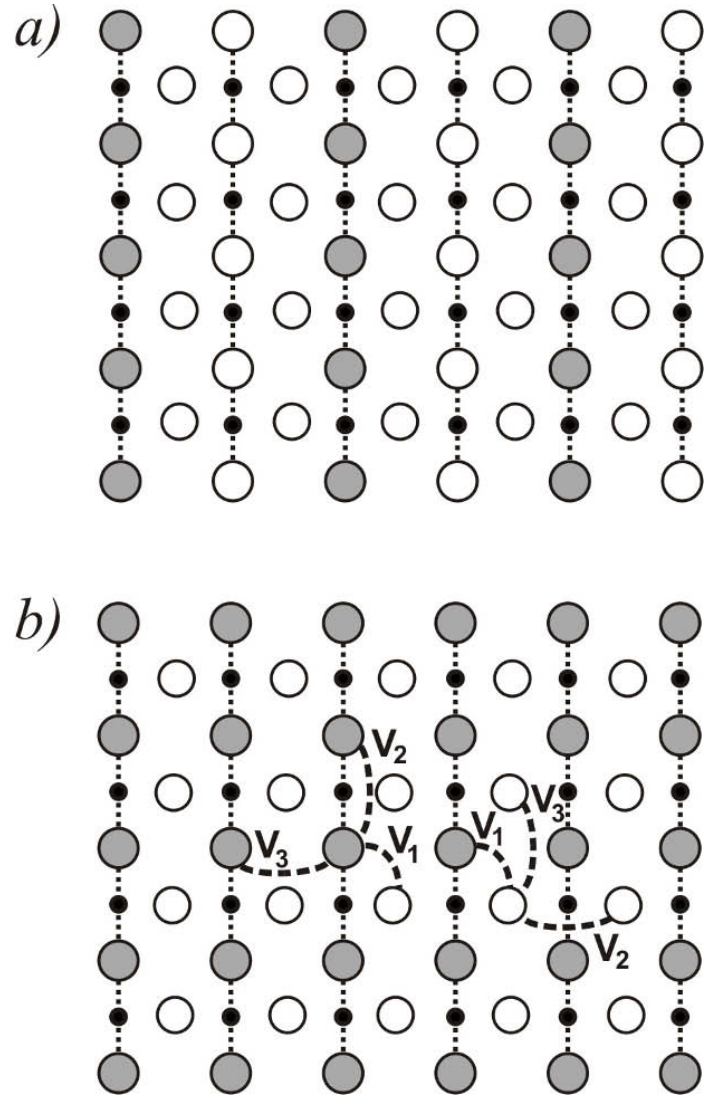

Figure 1. Arrangement of oxygen atoms in the basal planes of $\mathrm{YBa}_{2} \mathrm{Cu}_{3} \mathrm{O}_{6+x}$ material in a) Ortholl and b) Orthol structural phases, with the interactions of the ASYNNNI model shown. Small, black circles denote $\mathrm{Cu}$ atoms while the big circles represent the oxygen sites: shaded circle site occupied by an oxygen atom; empty circle - unoccupied.

the possible cluster microstates), and that the expression obtained is equivalent to the one found in Ref. [17]. for the chain length probability distribution of $\mathrm{Cu}-\mathrm{O}$ chains formed in the basal planes of YBCO material, studied in the frame of the ASYNNNI model. We will also show that in the case of the ASYNNNI model, the same chain length probability distribution applies along both of the next-nearest neighbor interactions present in the model.

\section{Chain length probability distribu- tion}

The cluster variation method is an approximate numerical method originally proposed by Kikuchi [22] for the calculation of phase diagrams of alloys. It has been reformulated several times which has enabled both its wider 
application [23-28], and contributed to an improved understanding of the method itself. Though the method yields mean field critical exponents, it is able to predict the values of the critical transition temperatures very accurately. While the $1 \mathrm{~d}$ Ising model can be treated exactly within the CVM formalism, in the 2D and 3D cases this method gives approximate results with an accuracy that is dependant on the size of the basic cluster. It has been shown that the approximate results obtained by the CVM method tend to the exact results as the size of the basic cluster increases [29].
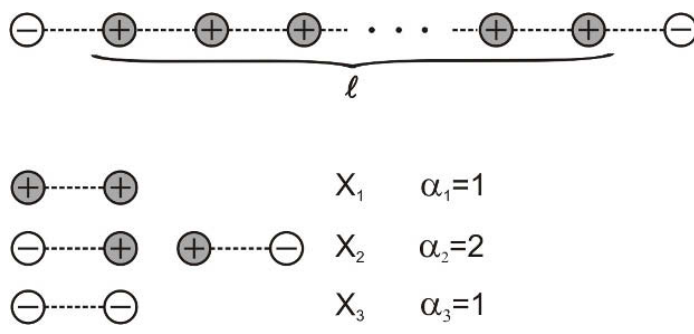

Figure 2. Chain fragment of length $l$ contained in the $1 \mathrm{~d}$ Ising chain, and the microstates of the pair cluster with corresponding probabilities and state degeneracies.

In the simple case of the ordinary 1d Ising model, which can be represented by an infinite Ising chain consisting of spins coupled by the nearest neighbor exchange interactions, it is sufficient to choose a pair of spins as a basic cluster for the CVM implementation. Possible microstates of the pair cluster and the corresponding probabilities and state degeneracies are shown at Fig. 2. We shall consider the full chain fragment of length $l$, within the infinite Ising chain, to consist of the sequence of $l$ "up" spins, $\sigma_{i}=+1$, (analogous to the $l$ sites occupied by an oxygen atom in the case of the $\mathrm{CuO}_{x}$ lattice), with end sites having "down" spins, $\sigma_{i}=-1$, (analogous to unoccupied sites of the lattice) as neighbours. The probability distribution of the full chain fragment can be constructed using the iterative procedure presented in the work of Vinograd et al. $[30,31]$. This procedure allows the probabilities of chain fragments of any length to be calculated as a function of pair and point cluster microstate probabilities. Appling this procedure, and employing the fact that the point cluster probabilities can be expressed through the pair cluster probabilities, the probability of a full chain fragment having length $l(0<l)$ can be written as a function of the pair cluster probabilities $x_{1}$ and $x_{2}$ :

$$
\begin{gathered}
p(l)=x_{2} \frac{x_{1}}{x_{1}+x_{2}} \frac{x_{1}}{x_{1}+x_{2}} \cdots \frac{x_{1}}{x_{1}+x_{2}} \frac{x_{2}}{x_{1}+x_{2}}= \\
=\frac{x_{2}^{2}}{x_{1}}\left(\frac{x_{1}}{x_{1}+x_{2}}\right)^{\prime} .
\end{gathered}
$$

Since we want these probabilities to fall between zero and 1 we normalize them according to the following normalizing condition:

$$
\sum_{l} p(l)=1
$$

Thus, we finally obtain true chain length probabilities:

$$
p(l)=\frac{x_{2}}{x_{1}+x_{2}}\left(\frac{x_{1}}{x_{1}+x_{2}}\right)^{l-1} .
$$

These normalized probabilities represent the fraction of the chains having length $l$ :

$$
p(l)=\frac{n(l)}{\sum_{k} n(k)},
$$

where $n(l)$ is the number of chain fragments of length, $l$, and $\sum_{k} n(k)$ is the total number of chain fragments in the system. Let us denote $c$, the concentration of up $(+1)$ spins (or oxygen atoms in the case of the basal plane lattice of YBCO material) in the chain, and let $n$ be the fraction of oxygen atoms situated at the end of the full chain fragment. These two variables can be expressed through the pair cluster configuration probabilities as follows:

$$
c=x_{1}+x_{2}, \quad n=2 x_{2} .
$$

Since every full chain fragment has two atoms residing at the chain ends, the total number of chain fragments will be proportional to half the number of the chain ends. Thus the following expression for the average chain length can be written:

$$
l_{a v}=\frac{2 c}{n}=\frac{x_{1}+x_{2}}{x_{2}} .
$$

After few manipulations the chain probability $p(l)$ can be expressed, as a function of $c$ and $T$, through a single parameter, the average chain length $l_{a v}(c, T)$ :

$$
p(l)=\frac{\left(1-l_{a v}\right)^{l-1}}{l_{a v}^{l}} .
$$

which is exactly the expression for the chain probability distribution which was shown in Ref. [17] to be valid for the ASYNNNI model at low temperatures.

Using expression (3) and the definition of the cluster microstate probabilities in the cluster variation method [32]:

$$
x_{l, i}=\frac{1}{Z_{l}} \exp \left(-E_{l . i} / k_{B} T\right),
$$



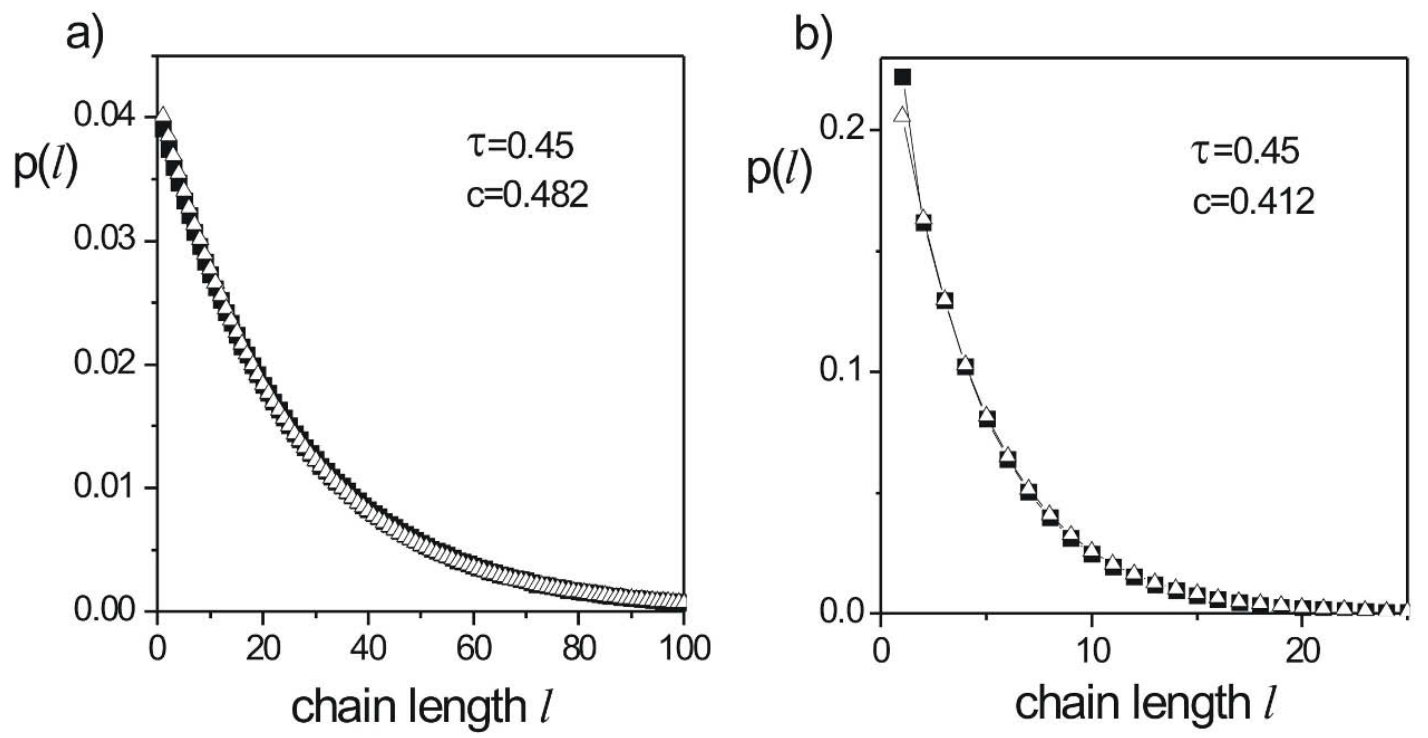

Figure 3. Values of the chain length probabilities $p(l)$ as a function of chain lengths $l$ along $V_{3}$ bonds of the ASYNNNI model presented for two different concentrations $c$ in the range of Orthol structural phase at $\tau=0.45 \approx 450 \mathrm{~K}$. Solid squares are points obtained by the MC numerical simulations while the open triangles represent results found using the CVM method and expression (3).

where $E_{l, i}$ is the energy of the cluster $l$ in its $i$-th cluster microstate, and $Z_{l}$ is its partition function, the chain length probability can be cast in the following form:

$$
p(l)=r(1-r)^{l-1},
$$

where $r=\left(1+e^{\beta\left(E_{2}-E_{1}\right)}\right)^{-1}, \beta=k_{B} T$. In the case of the $1 \mathrm{~d}$ Ising model with ferromagnetic coupling $J$, we have $E_{2}-$ $E_{1}=2 J$ with expression (9) being equal to the probability distribution of the number of spins in the "up" domain in an infinite Ising chain as reported in Ref. [19].

\section{Numerical simulations and dis- cussion}

In the previous section the chain length probability distribution of the $1 \mathrm{~d}$ Ising chain was expressed through the pair cluster probabilities $x_{i} \quad(i=1,2)$, the values of which determine the microstate of the complete Ising chain. We have shown that expression (3) is equivalent to that used in the ASYNNNI model for the probabilities, $p(l)$, of the $\mathrm{Cu}-\mathrm{O}$ chains formed along $V_{2}$ bonds. The equivalency of the two expressions is considered to be a consequence of the previously established isomorphism between this and the $1 \mathrm{~d}$ Ising model. However, the pair cluster probabilities, which are to be used in expression (3) to calculate the chain fragment probabilities of the ASYNNNI model formed along $V_{2}$ bonds, are not the same as those obtained from the simple pair approximation of the CVM method when applied to the $1 \mathrm{~d}$ Ising chain coupled by $V_{2}$ interaction. Instead, when expression (3) is applied to the ASYNNNI model, the probabilities $x_{1}$ and $x_{2}$ are taken as the equilibrium cluster probabilities within some higher order CVM approximation appropriate for the ASYNNNI model investigation. This higher order approximation will certainly include basic clusters much larger than the pair clusters sufficient to treat the $1 \mathrm{~d}$ Ising model. As a result, the pair cluster probabilities will include information on the influence that the surrounding spins have on the interaction of two spins incorporated in the chain. Thus, in terms of the chain length probabilities, the ASYNNNI model can be considered, equivalent to the 1d Ising model in which spins are coupled by some effective pair interaction which incorporates the effect of the surrounding sites on the interaction energy of the spin pair considered, as well as the bare pair spin interaction of the ASYNNNI model. This equivalency can be assumed to exist along each of the two next-nearest neighbour pair interactions included in the ASYNNNI model, but it is more evident along the attractive $V_{2}$ interaction which induces the formation of long chain fragments.

It is also important to note that, from expression (6) and the definition of the variable $r$, we can derive the relationship, 
a)

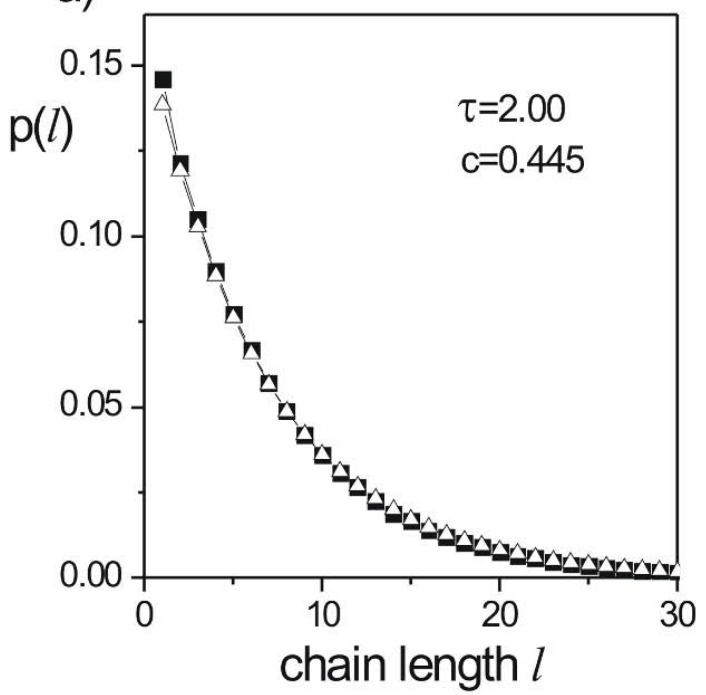

b)

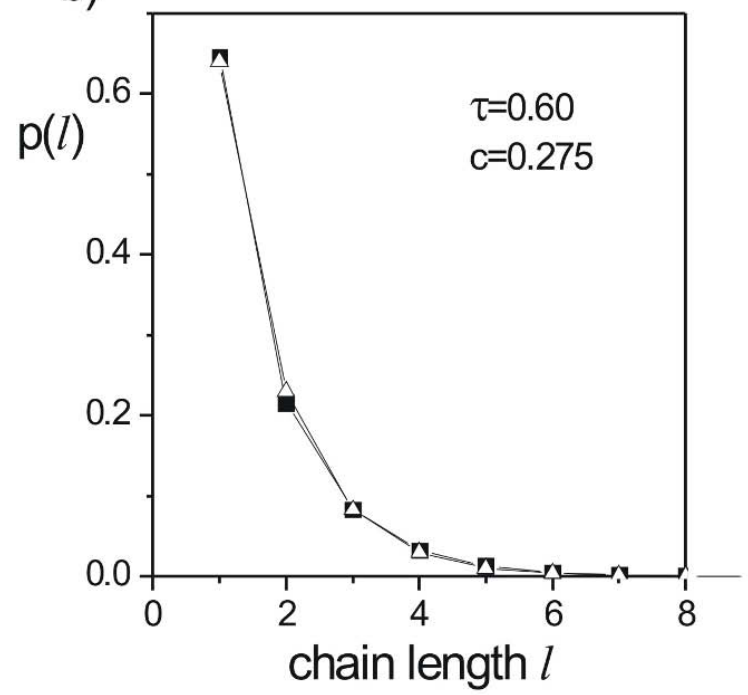

Figure 4. Dependence of the chain length probabilities $p(l)$ on the chain lengths $l$ obtained by MC (solid squares) and CVM (open triangles) showing the validity of expression (3) at high temperatures a) and in the disordered tetragonal structural phase b).

$l_{a v}=1 / r$. Since the variable $r$ is, in the case of the $1 \mathrm{~d}$ Ising chain with ferromagnetic interaction $J>0$, bounded from above by $1 / 2$, it follows that the ASYNNNI model can be considered equivalent to the ferromagnetically coupled Ising model only when the condition $l_{a v}>2$ is met.

To prove the validity of expression (3) for the ASYNNNI model, the chain length probability distribution, $p(l)$, was calculated by using the cluster variation method $(4,5-$ points cluster approximation) and expression (3), and then compared with the value of $p(l)$ calculated directly in Monte Carlo simulations of the ASSYNNI model (applying the standard Metropolis algorithm). The Hamiltonian of the ASYNNNI model can be expressed by the following equation:

$$
H=-\mu \sum_{i} \sigma_{i}+V_{1} \sum_{N N} \sigma_{i} \sigma_{j}+V_{2} \sum_{N N N^{\prime}} \sigma_{i} \sigma_{j}+V_{3} \sum_{N N N} \sigma_{i} \sigma_{j}
$$

where $\sigma_{i}$ is the Ising spin, which can take the value +1 or -1 depending on the site, $i$ occupancies, $\mu$ is the chemical potential representing external field, and $V_{1}, V_{2}$ and $V_{3}$ are the model interactions (see Fig. 1): $V_{1}$ and $V_{3}$ are repulsive, while $V_{2}$ is attractive. The first sum goes over all sites and second one is taken over all nearest neighbours. The third summation is performed over all next nearest neighbour sites along $V_{2}$ bond, while the last one is calculated over next nearest neighbours coupled via $V_{3}$ interaction. The values of the interaction parameters used were those obtained by Sterne and Wille from the first principle calculations using the linear-muffin-tin orbital method: $V_{1}=6.9 \mathrm{mRy}, V_{2}=-2.4 \mathrm{mRy}$ and $V_{3}=1.1 \mathrm{mRy}$ [33]. In order to determine the probabilities, $p(l)$, the number of chains of length $l$, and total number of chains were calculated for every lattice microstate generated in every accepted Monte Carlo step of the simulation; the maximal chain length was determined by the size of the system.

The results of the numerical simulations are presented in Fig. 3. The figure shows the probability distribution of chains extending along $V_{3}$ interactions as a function of chain length $l$ calculated at the reduced temperature $\tau=k_{B} T / V_{1}=0.45(T \approx 450 \mathrm{~K})$. Results are shown for two different oxygen concentrations $c(x=2 c)$ in the range of the Ortho I structural phase of the $\mathrm{YBa}_{2} \mathrm{Cu}_{3} \mathrm{O}_{6+x}$ material. Though some discrepancies are present for the shortest chains, it can be seen that the results obtained from Monte Carlo simulations performed on a system of $200 \times 200$ spins are in excellent agreement with those obtained using the 4,5-points cluster approximation of the CVM method employing the expression (3). Monte Carlo numerical simulations were also performed at higher temperatures, up to $2000 \mathrm{~K}$ in the range of the Ortho I structural phase (Fig. 4a); once again the results showed excellent agreement with those obtained by the CVM method. Furthermore, relation (3) was also found to be valid in the disordered tetragonal phase, though in this structural 
phase the chains become rather short (Fig. 4b).

\section{Conclusion}

In this study the chain length probabilities of the 1d Ising model were expressed through the pair cluster probabilities. The same expression was used to calculate chain length probabilities in the frame of the $2 \mathrm{~d}$ ASYNNNI model, and the results are compared to those obtained by numerical Monte Carlo simulations. It was argued that the ASYNNNI model can be considered equivalent to a $1 \mathrm{~d}$ Ising model in which the coupling is modeled as a pair cluster interaction with a spin pair interaction energy that includes a contribution from both the interaction between nearest neighbour spins, and the interaction of surrounding spins with the spin pair. In general, the equivalency of the two models can be assumed to exist along any of the next-nearest neighbor pair interactions included in the ASYNNNI model, however, when the ASYNNNI model is applied to the problem of oxygen ordering in the basal planes of $\mathrm{YBa}_{2} \mathrm{Cu}_{3} \mathrm{O}_{6+x}$ material, it is most evident along the direction of $\mathrm{Cu}-\mathrm{O}$ chain formation, i.e. along the $V_{2}$ coupling.

\section{Acknowledgments}

This work was supported by the Republic of Serbia Ministry of Sciences under the project No. 141014.

\section{References}

[1] C. Chailloutet et al., Phys. Rev. B 36, 7118 (1987)

[2] M.V. Zimmerman et al., arXiv:cond-mat/9906251

[3] J.D. Jorgensen et al., Physica C 167, 571 (1990)

[4] B.W. Veal et al., Phys. Rev. B 42, 6305 (1990)

[5] G.V. Uimin et al., Physica C 192, 481 (1992)

[6] G. Uimin, Phys. Rev. B 50, 9531 (1996)

[7] P. Gawiec, D.R. Grempel, A.-C. Riiser, H. Haugerud, G. Uimin, Phys. Rev. B 53, 5872 (1996)
[8] P. Gawiec, D.R. Grempel, G. Uimin, J. Zittarz, Phys. Rev. B 53, 5880 (1996)

[9] H. Haugerud, G. Uimin, W. Selke, Physica C 275, 93 (1997)

[10] D.M. Bubb, J.F. Federici, J. Phys.-Condens. Matter 12, L261 (2000)

[11] G. Uimin, J. Rossat-Mignod, Physica C 199, 251 (1992)

[12] D. de Fontaine, G. Ceder, M. Asta, Nature 343, 544 (1990)

[13] D. Monster, P.A. Lindgard, N.H. Andersen, Phys. Rev. B 60, 110 (1999)

[14] D. Monster, P.A. Lindgard, N.H. Andersen, Phys. Rev. B 64, 224520 (2001)

[15] V.M. Mati_, M. Mili_, N.DJ. Lazarov, Physica C 422, 1 (2005)

[16] S. Maennsteadt, G.S. Pawley, Phys. Rev. B 62, 4169 (2000)

[17] V.M. Matić, N.Dj. Lazarov, V. Spasojević, M. Milić, V. Kusigerski, Physica C 421, 49 (2005)

[18] V. M. Matić, N.Dj. Lazarov, Physica C 443, 49 (2006)

[19] S.I. Denisov, P. Hanggi, Phys. Rev. E 71, 046137 (2005)

[20] M.B. Yilmaz, F.M. Zimmermann, Phys. Rev. E 71, 026127 (2005)

[21] V.M. Matić et al., Phys. Rev. B 62, 107 (2000)

[22] R. Kikuchi, Phys. Rev. B 81, 988 (1951)

[23] J. Hijmans, J. de Boer, Physica 21, 471 (1955)

[24] J.A. Barker, Proc. R. Soc. A 216, 45 (1953)

[25] T. Morita, J. Phys. Soc. Jpn. 12, 753 (1957)

[26] T. Morita, J. Math. Phys. 13, 115 (1972)

[27] G. An, J. Stat. Phys. 52, 727 (1988)

[28] T. Morita, J. Stat. Phys. 59, 819 (1990)

[29] A.G. Schlijper, Phys. Rev. B 27, 6841 (1983)

[30] V.L. Vinograd, S.K. Saxena, A. Putnis, Phys. Rev. B 56, 11493 (1997)

[31] V.L. Vinograd, Am. Mineralog. 84, 311 (1999)

[32] V.M. Matić, S. Milosević, Physica A 262, 215 (1999)

[33] P.A. Sterne, L.T. Wille, Physica C 162, 223 (1989) 